\title{
Oncologists' and cancer patients' views on whole-exome sequencing and incidental findings: results from the CanSeq study
}

\author{
Stacy W. Gray, MD, $\mathrm{AM}^{1-3}$, Elyse R. Park, PhD, $\mathrm{MPH}^{4}$, Julie Najita, $\mathrm{PhD}^{5}$, \\ Yolanda Martins, $\mathrm{PhD}^{1}$, Lara Traeger, $\mathrm{PhD}^{4}$, Elizabeth Bair, BA ${ }^{1}$, Joshua Gagne, $\mathrm{MA}^{1}$, \\ Judy Garber, MD, $\mathrm{MPH}^{1-3}$, Pasi A. Jänne, $\mathrm{MD}, \mathrm{PhD}^{1-3}$, Neal Lindeman, $\mathrm{MD}^{2,6}$, \\ Carol Lowenstein, MBA ${ }^{1}$, Nelly Oliver, PhD ${ }^{1}$, Lynette Sholl, MD2,6, \\ Eliezer M. Van Allen, MD ${ }^{1-3,7}$, Nikhil Wagle, $\mathrm{MD}^{1-3,7}$, Sam Wood, BA ${ }^{1}$, \\ Levi Garraway, MD, $\mathrm{PhD}^{1-3,7}$ and Steven Joffe, MD, $\mathrm{MPH}^{8}$
}

Purpose: Although targeted sequencing improves outcomes for many cancer patients, it remains uncertain how somatic and germ-line whole-exome sequencing (WES) will integrate into care.

Methods: We conducted surveys and interviews within a study of WES integration at an academic center to determine oncologists' attitudes about WES and to identify lung and colorectal cancer patients' preferences for learning WES findings.

Results: One-hundred sixty-seven patients ( $85 \%$ white, $58 \%$ female, mean age 60 ) and 27 oncologists (22\% female) participated. Although oncologists had extensive experience ordering somatic tests (median $100 /$ year), they had little experience ordering germ-line tests. Oncologists intended to disclose most WES results to patients but anticipated numerous challenges in using WES. Patients had moderately low levels of genetic knowledge (mean 4 correct out of 7). Most patients chose to learn results that could help select a clinical trial, pharmacogenetic and positive prognostic results, and results suggesting inherited predisposition to cancer and treatable noncancer conditions (all $\geq 95 \%$ ). Fewer chose to receive negative prognostic results $(84 \%)$ and results suggesting predisposition to untreatable noncancer conditions (85\%).

Conclusion: The majority of patients want most cancer-related and incidental WES results. Patients' low levels of genetic knowledge and oncologists' inexperience with large-scale sequencing present challenges to implementing paired WES in practice.

Genet Med advance online publication 11 February 2016

Key Words: cancer; incidental findings; return of results; sequencing

\section{INTRODUCTION}

Exome and genome sequencing are disruptive technologies that may transform clinical practice. Physicians and patients will confront vast amounts of complex and uncertain data, including incidental findings unrelated to the testing indication. If recommendations regarding obligations to return incidental genomic results ${ }^{1,2}$ are adopted, then patients will also face decisions about the types of genomic information that they would like to receive.

Given substantial uncertainty regarding how to best deliver exome- and genome-guided medical care, it is imperative that we understand how whole-exome sequencing (WES) will alter clinical practice and anticipate the challenges that providers and patients will face. Oncology is an ideal setting in which to explore clinical sequencing because cancer is often driven by genomic changes. Targeted germ-line (normal tissue) and somatic (tumor) DNA sequencing, used separately, have dramatically improved outcomes in some high-risk ${ }^{3-6}$ and cancer patient subpopulations, ${ }^{7-10}$ and larger gene panels are already used in practice. ${ }^{11,12}$ Sequencing's power increases when somatic and germ-line DNA are sequenced in parallel, because paired sequencing unequivocally distinguishes somatic from germ-line alterations $s^{13,14}$ and can uncover previously unsuspected inherited cancer risk. ${ }^{15}$

To inform the debate regarding how to implement cancer-related WES in the best way, we initiated a prospective study to explore how introducing WES into care might affect cancer patients and oncologists. This article reports findings from baseline surveys and interviews with patients and physicians. We hypothesized that patients would want to receive information about all potentially informative somatic and germ-line genomic alterations, and that oncologists would

${ }^{1}$ Department of Medical Oncology, Dana-Farber Cancer Institute, Boston, Massachusetts, USA; ${ }^{2}$ Harvard Medical School, Boston, Massachusetts, USA; ${ }^{3}$ Department of Medicine, Brigham \& Women's Hospital, Boston, Massachusetts, USA; ${ }^{4}$ Massachusetts General Hospital, Boston, Massachusetts, USA; ${ }^{5}$ Department of Biostatistics and Computational Biology, Dana-Farber Cancer Institute, Boston, Massachusetts, USA; ${ }^{6}$ Department of Pathology, Brigham \& Woman's Hospital, Boston, Massachusetts, USA; ${ }^{7}$ Broad Institute, Cambridge, Massachusetts, USA; ${ }^{8}$ Department of Medical Ethics and Health Policy, University of Pennsylvania Perelman School of Medicine, Philadelphia, Pennsylvania, USA.

Correspondence: Stacy W. Gray (stacyw_gray@dfci.harvard.edu) 
anticipate numerous clinical, psychosocial, and ethical challenges as they prepare to evaluate and disclose WES results.

\section{PARTICIPANTS AND METHODS Study setting, dates, and participants}

The Dana-Farber Cancer Institute (DFCI) CanSeq study, launched in February 2013, is a single-arm prospective study of the integration of paired WES into clinical care. The eligible population for the CanSeq clinician study included all medical oncologists in the Thoracic and Gastrointestinal Oncology Centers who care for lung or colorectal adenocarcinoma patients. Two oncologists are study coinvestigators and were excluded from baseline survey and interview participation; one oncologist declined to participate in the CanSeq study. Because CanSeq's goal is to understand both patients' and physicians' experiences with WES, only patients of participating oncologists were eligible.

The eligible population for the CanSeq patient study includes patients who (i) have stage IV lung or colorectal adenocarcinoma, (ii) consent to companion genotyping protocols (to allow variant confirmation by a complementary technology), (iii) have a life expectancy of at least 6 months, (iv) have an Eastern Cooperative Oncology Group performance status of zero or one, (v) have sufficient tumor DNA for WES, (vi) have a treating oncologist who is participating in the study, (vii) speak English, (viii) consent to participation, and (iv) receive ongoing care at DFCI. All study activities were approved by the DFCI Institutional Review Board, and both patient and physician participants gave written informed consent.

\section{Study procedures}

Oncologist survey and interview procedures. The oncologist survey was offered at the time of enrollment. Electronic reminders were sent out at 1-week intervals until survey completion. After three contacts, a study investigator contacted nonresponders to encourage participation. A sub-sample of oncologists, stratified by gender and academic rank, was invited to participate in individual interviews. Interviews were conducted in person or by telephone within approximately 1 month of physician enrollment. Interviews were audiorecorded and transcribed for analysis.

Patient consent and survey procedures. After confirming patient eligibility with the treating physician, clinical research assistants approached patients during routine clinic visits to offer participation. At the time of consent, patients were asked to report their preferences for the receipt of cancer-related and noncancer-related WES findings. Immediately following consent, patients were asked to complete the baseline survey on a computer tablet or on paper. Patients who did not complete the survey in the clinic could complete it at home. Reminder letters/e-mails were sent to nonresponders 2, 4, and 6 weeks after consent. The clinical research assistants also approached patients during subsequent clinic appointments to facilitate survey completion. We paused the reminders if medical record review or provider message identified an acute illness or hospitalization. Participants were considered nonresponders if they had not completed the baseline survey within 2 months of enrollment or by the time of result reporting.

\section{Measures and domains}

Physician survey measures and interview domains. The physician survey contained questions related to experience with somatic and germ-line testing during the prior year, attitudes about the return of sequencing results, confidence in the ability to perform relevant tasks (e.g., interpret data, explain concepts to patients, make treatment recommendations, provide psychosocial support, obtain informed consent), and sociodemographic and practice characteristics (Physician Baseline Survey Instrument, Supplementary Material S1 online).

We created a measure to assess oncologists' attitudes about the return of genome results. The three-item measure asked how strongly oncologists agreed or disagreed with limiting return of results to those with clinical utility (evidence demonstrates that actions based on the results can change patient management decisions and improve net health outcomes), returning results with clinical validity (evidence of an established relationship between genotype and phenotype) but not utility, and returning all genomic sequencing results. The somatic genomic confidence measure was adapted from our prior work ${ }^{16}$ to include two additional items related to the oncologist's ability to identify consultants with expertise in integrating somatic genomic information into patient care and to provide psychosocial support related to coping with somatic information with adverse prognostic information. We adapted Nippert's germ-line confidence scale ${ }^{17}$ for the cancer context. We elicited intentions to disclose WES information using short vignettes describing an adult patient with a metastatic solid tumor. The hypothetical patient had undergone somatic and germ-line WES performed in a clinically certified laboratory, was receiving first-line chemotherapy, had an Eastern Cooperative Oncology Group performance status of 0 or 1 , had indicated that he or she would like to be told about all clinically valid results, and had biological children. We asked about intentions to disclose somatic predictive alterations (i.e., that could be targeted with a drug available through a phase II clinical trial or that is approved by the US Food and Drug Administration for a different cancer type) and somatic prognostic alterations (positive and negative). We also asked about intentions to disclose germ-line cancer risk alterations (with and without available risk-reduction strategies), pharmacogenetic polymorphisms (cancer and noncancer-related), alterations that conferred increased risk of developing a noncancer condition (with and without available risk-reduction strategies), and carrier status. We conducted cognitive testing (structured survey review and feedback elicitation) of the draft survey instrument with five oncologists in disease centers other than gastrointestinal and thoracic, revised the survey, and finalized the survey. The survey was administered on paper and took $\leq 10$ minutes to complete. 
The oncologist interviews were developed based on the researchers' prior experiences and the published literature (Physician Baseline Interview Guide, Supplementary Material S2 online). We pilot-tested the interview guide with two oncologists, revised it, and finalized it. The interview took $\sim 30$ minutes and covered the following: expectations related to WES; anticipated benefits, risks, and challenges of using WES in clinical practice; and intentions to disclose results to patients. Data were collected until thematic saturation was achieved. Interviews were transcribed, reviewed for accuracy and completeness, and uploaded into NVivo 10 (QSR, Doncaster, Victoria, Australia).

Patient measures. The patient consent form included nine questions to elicit patients' preferences for the disclosure of somatic WES results (results that could be used to identify possible clinical trials; positive/negative prognostic results) and germ-line WES results (cancer risk; cancer and noncancer pharmacogenetic; risk of developing treatable and nontreatable conditions other than cancer; and carrier status) (Patient Consent Preferences, Supplementary Material S3 online). The patient baseline survey instrument (Supplementary Material S4 online) included validated measures to assess patients' attitudes about undergoing a genetic test, ${ }^{18}$ experience with genetic testing, ${ }^{19}$ genetic knowledge, ${ }^{20,21}$ subjective numeracy, ${ }^{22}$ health literacy, ${ }^{23}$ self-reported Eastern Cooperative Oncology Group performance status, ${ }^{24,25}$ quality of life, ${ }^{26}$ decision-making preferences, ${ }^{27}$ and sociodemographic characteristics. We cognitively tested the draft patient consent questions and survey instrument with five patients with advanced lung and colorectal cancer and then revised and finalized the instruments.

\section{Analyses}

Surveys. The aims of the oncologist survey were to describe oncologists' attitudes about WES disclosure, intentions to disclose WES results, somatic and germ-line genomic confidence, as well as the frequency of baseline genomic testing. The aims of the patient consent items and baseline survey were to describe patients' preferences for the return of WES results and their genetic knowledge. In exploratory analyses, we evaluated the associations between patient characteristics and (i) attitudes about getting a genetic test and (ii) preferences regarding receipt of somatic and germline sequencing results. Attitudes about undergoing a genetic test were evaluated as a dichotomous outcome $(<2 / 5$ very positive vs. $\geq 2 / 5$ ). Subjects who indicated a preference for return of all three types of somatic results and all six types of germ-line results were counted as having high preference for somatic results and germ-line results, respectively (high preference vs. other). We explored the associations between attitudes/preferences and age, gender, cancer type, education, genetic knowledge, and attitudes about undergoing a genetic test (preferences only) in univariate analyses. Independent variables with univariate $P$ values less than 0.20 were included in a multivariable model. Relative risks estimates and 95\% confidence intervals (CIs) were determined using a modified Poisson regression with robust variance estimates. ${ }^{28}$

Interviews. Two team members independently coded qualitative data using NVivo 10 to develop a coding framework guided by the interview guide domains. An iterative process of structured coding ensued, with discrepancies resolved through discussion and comparison to the raw data; a final $\kappa$ of 0.88 was achieved.

\section{RESULTS}

\section{Participant characteristics}

All 27 participating oncologists completed the baseline physician survey. One hundred sixty-seven patients indicated preferences at the time of consent, and 153 patients completed the patient baseline survey (response rates 100 and 92\%, respectively; Supplementary Figure S1 online). Patient and oncologist characteristics are shown in Table 1.

Oncologists. Oncologists spent the majority of time in patient care and research. They reported ordering or interpreting a median of 100 somatic tests per year (interquartile range: 40 100) and two germ-line cancer predisposition tests per year (interquartile range: $0-10$; Supplementary Table S1 online). Few oncologists had ordered or interpreted germ-line tests unrelated to cancer $(n=4)$, cancer-related pharmacogenetic tests $(n=9)$, noncancer pharmacogenetic $(n=2)$, or tests to identify carrier status $(n=2)$.

Patients. Thirteen percent of patients reported having had genetic testing. Patients had positive attitudes about undergoing a genetic test (mean 1.3 on a $1-5$ point scale, with $1=$ most positive). No patient characteristics were associated with positive attitudes about undergoing a genetic test.

\section{Physicians' genomic confidence, disclosure philosophy, and intentions to disclose WES results}

Most oncologists were very or moderately confident in their ability to perform many somatic and germ-line genomic tasks (Supplementary Table S2 online). Oncologists were less confident in their abilities to provide psychosocial support related to negative prognostic results and to perform activities related to cancer risk testing (i.e., provide pretest counseling, obtain informed consent, and provide psychosocial support). Oncologists' attitudes about the return of genomic test results varied (Figure 1). Seventy-eight percent supported disclosure if WES results established clinical validity and $67 \%$ did not support limiting return of results to those with established clinical utility. Fifty-two percent agreed that patients should be offered as many sequencing results as they want, including raw sequencing data. When asked about disclosure intentions, most oncologists said that they would disclose somatic and germline WES information (Table 2). Some oncologists reported reluctance to disclose somatic results that were in a pathway targeted by an agent that is approved by the Food and Drug 
Table 1 Physician and patient characteristics

\begin{tabular}{lc} 
Physician characteristics & $\begin{array}{c}\text { Frequency (\%), } \\
(\boldsymbol{n}=\mathbf{2 7})\end{array}$ \\
\hline Program & 52 \\
GI & 48 \\
Thoracic & \\
Principal investigator & 59 \\
Clinical trials & 44 \\
Translational research & 15 \\
Basic science & 22 \\
Outcomes/health services or &
\end{tabular}

cancer epidemiology

\begin{tabular}{lcc}
$\begin{array}{l}\text { Gender } \\
\text { Male }\end{array}$ & 78 & \\
Female & 22 & \\
\hline & Median & IQR \\
\hline $\begin{array}{l}\text { Year fellowship was completed } \\
\text { Number of unique patients seen }\end{array}$ & 2007 & $1999-2011$ \\
per month & 50 & $28-70$ \\
Percent of professional time spent in: & & \\
$\quad$ Patient care & 40 & $30-75$ \\
Research & 40 & $19-60$ \\
Teaching & 5 & $5-10$ \\
Administration & 5 & $0-15$
\end{tabular}

\begin{tabular}{lc}
\hline Patient characteristics & $\begin{array}{c}\text { Frequency }(\%), \\
(\boldsymbol{n}=167)^{\mathrm{a}}\end{array}$ \\
\hline $\begin{array}{l}\text { Age at consent, mean (SD) } \\
\text { Gender }\end{array}$ & $59.8(12.0)$ \\
$\quad$ Female & $97(58)$ \\
Cancer & \\
Lung & $89(53)$ \\
Colorectal & $78(47)$
\end{tabular}

Race $^{\text {b }}$

$\begin{array}{lr}\text { White } & 130(85) \\ \text { Nonwhite } & 19(13)\end{array}$

\begin{tabular}{lc}
$\begin{array}{l}\text { Hispanic/Latino } \\
\text { Education }^{\mathrm{b}} \\
\text { College graduate or higher }\end{array}$ & $3(2)$ \\
Overall health, mean (SD) ${ }^{\mathrm{b}, \mathrm{c}}$ & $68(44)$ \\
$\begin{array}{l}\text { Prior genetic testing } \\
\text {, d }\end{array}$ & $5.2(1.1)$ \\
Yes & $20(13)$ \\
No & $122(80)$ \\
Do not know & $11(7)$ \\
\hline $\begin{array}{l}\text { Attitude toward genetic testing, } \\
\text { mean (SD) }\end{array}$ & $1.3(0.7)$ \\
\hline
\end{tabular}

\section{$\mathrm{Gl}$, gastrointestinal; IQR, interquartile range.}

aPercentages may not add to $100 \%$ due to missing responses and/or rounding. ${ }^{b} n=153$ who completed baseline survey. 'Seven-point scale from very poor

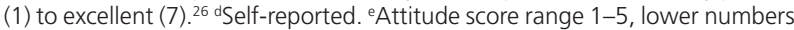
correspond to more positive attitudes. ${ }^{18}$

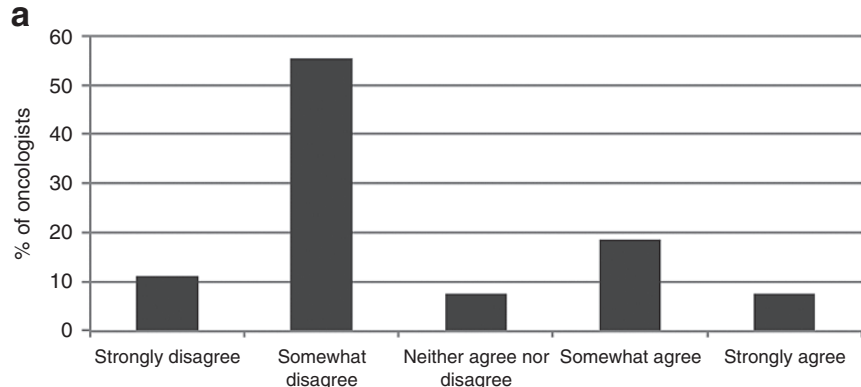

Clinical utility: Patients should only be offered their genomic sequence results if evidence demonstrates that actions based on the results can change patient management decisions and improve net health outcomes

b

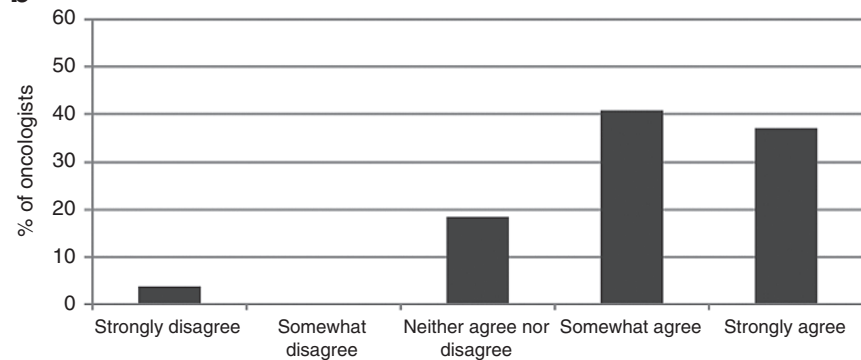

Clinical validity: Patients should be offered genomic sequence results for which there is an established relationship between genotype and phenotype, even if the results do not alter management decisions or improve net health outcomes

C

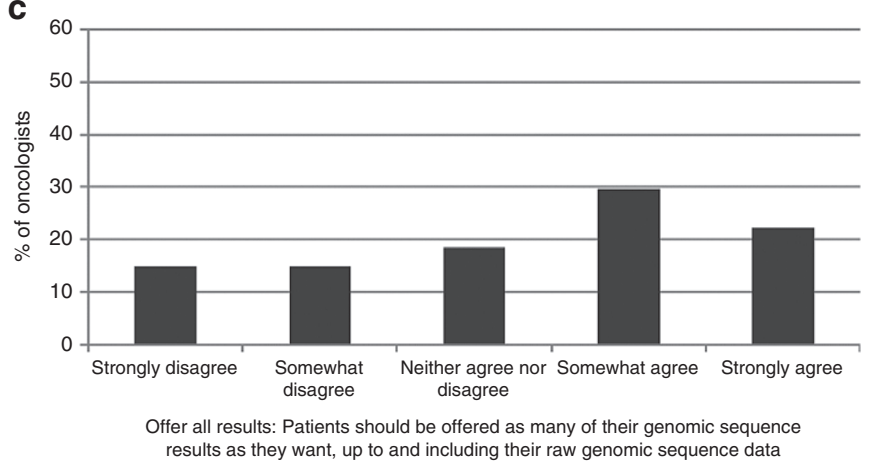

Figure 1 Oncologists' attitudes regarding return of genomic test results $(\boldsymbol{n}=\mathbf{2 7})$. Return based on (a) clinical utility, (b) clinical validity, and (c) all results.

Administration for another tumor type, negative prognostic results, pharmacogenetic results, results related to nontreatable conditions, and carrier status.

\section{Physicians' anticipated challenges in integrating WES into} practice and disclosure intention themes

Qualitative interviews generally reinforced the survey findings (Supplementary Results S1 online). Oncologists anticipated various challenges in using WES (Table 3). Physicians reported that they generally intended to disclose somatic and germ-line findings (Interview Themes: Supplementary Table S3 online). All physicians reported an intention to disclose predictive somatic findings; many also intended to disclose prognostic information. Some oncologists expressed reluctance to disclose germ-line information related to the risk of developing cancer 
Table 2 Oncologists' intentions to disclose somatic and germ-line WES findings to patient $(n=27)$

\begin{tabular}{|c|c|c|c|c|c|}
\hline & $\begin{array}{c}\text { Definitely } \\
\text { disclose (\%) }\end{array}$ & $\begin{array}{c}\text { Probably } \\
\text { disclose (\%) }\end{array}$ & $\begin{array}{l}\text { Probably not } \\
\text { disclose (\%) }\end{array}$ & $\begin{array}{c}\text { Definitely not } \\
\text { disclose (\%) }\end{array}$ & $\begin{array}{c}\text { Unsure } \\
(\%)\end{array}$ \\
\hline \multicolumn{6}{|l|}{ Sequencing of tumor DNA identifies a somatic alteration that is: } \\
\hline Predictive: targeted in a phase II clinical trial & 81 & 19 & - & - & - \\
\hline Predictive: targeted by an agent that is FDA-approved for a different cancer & 59 & 30 & 7 & - & 4 \\
\hline Prognostic: favorable & 56 & 44 & - & - & - \\
\hline Cancer risk alteration: treatable & 81 & 19 & - & - & - \\
\hline Cancer risk alteration: not treatable & 30 & 44 & 15 & - & 11 \\
\hline Pharmacogenetic polymorphism: cancer-related & 74 & 15 & 11 & - & - \\
\hline Pharmacogenetic polymorphism: noncancer-related & 56 & 0 & 15 & - & - \\
\hline
\end{tabular}

Predictive: The alteration/pathway may be targeted by a therapeutic agent. Prognostic: The alteration confers a favorable or unfavorable prognosis.

FDA, US Food and Drug Administration; WES, whole-exome sequencing.

Table 3 Oncologists' anticipated challenges when using WES in clinical practice $(n=19)$

Themes

Ability to distill data and identify actionable findings

Large data volume and few actionable results

Determining what to disclose to patients

Managing patient expectations

Need for physician education Managing and dis
incidental findings

Managing patient and family emotional response to WES disclosure

\section{Examples}

- "The biggest challenge is swimming through the noise. You're going to get a lot of information. In the absence of [obviously actionable information], if you're getting a lot of minor, rare mutations, what do we do with that information?"

- "The biggest challenge is that there will be a lot of information and we'll have relatively few therapies at this moment that will be driven by the results."

- "How much do you share with patients?...Are patients going to be overwhelmed with the volume of information?...I think it very much depends not only on the output but how we present it to patients, and how much we decide to share."

- "Many patients think that whole exome sequencing will solve [the question] of which drugs to give to patients and [will help us] come up with a miracle cure, but in fact... there are a lot of [mutations] that we cannot act on."

- "You have to be cognizant that patients will want something tangible from this and they may not get it...and so we have to be good at explaining [this]."

- "You have to keep up with the literature and understand what these sequences could potentially mean, and that takes a certain amount of education on our part."

- " "It's coming whether we want it or not, so we may as well learn it! It's a fine opportunity."

- "Because of all of the uncertainty of findings or potentially negative findings that create anxiety, I don't think that's information that will be useful or helpful to patients, and I'd be really hesitant to discuss it at all."

- Appreciating the anxiety that patients have about this, [we need to be] more discrete about which results we talk about. A less important result may lead to a lot of stress and so I would have a very high threshold to tell anyone that there is something we found in their germ line."

- "If you find something, you have to really be able to be able to communicate this stuff in a way that will be compassionate and thoughtful and will not overwhelm a patient."

- "Some [patients] will be fraught with anxiety over whatever result they get. And some will want to understand every gene that they see a base pair off on. I think there are going to be different needs for different people."

- " "In the cancer world, we are normally dealing in the somatic realm, which we have a lot of experience doing. " But especially discussing small effects in the germ line, it may be challenging to do."

- " 'I'm not very experienced in counseling people about germ-line testing and identification of risk for diseases or familiar syndromes."

WES, whole-exome sequencing 
and noncancer conditions and noncancer pharmacogenetic alterations. Several oncologists mentioned that such disclosures should involve another physician or a genetic counselor. Determinants of oncologists' intentions to disclose WES findings included patients' preferences and performance status, the physician's knowledge, and the "actionability" of findings (e.g., the availability of relevant targeted therapies for somatic alterations or relevant risk reduction interventions for germ-line alterations). Some oncologists believed that patients have a right to know all information learned.

\section{Patients' genetic knowledge and preference for learning WES results}

Patients had moderately low genetic knowledge, with a mean score of 4 correct out of 7 (Supplementary Figure S2 online). A sizable minority did not know that genetic testing can be used to evaluate cancer risk, that fathers can pass on genetic conditions, and that people who have mutations do not always develop disease.

Almost all patients chose to learn most cancer-related, pharmacogenetic, and carrier status findings (Figure 2). Slightly fewer patients opted to receive negative prognostic results $(84 \%)$ and information about the risk of developing an untreatable noncancer condition (85\%). After adjusting for gender, patients with less positive attitudes about undergoing a genetic test were less likely than those with very positive attitudes to indicate a high preference for the return of somatic ( $65 \mathrm{vs.} 86 \%$, adjusted risk ratio (RR): $0.89,95 \%$ CI: $0.80-0.98, P=0.02$ ) and germ-line (65 vs. $86 \%$, adjusted RR: $0.89,95 \%$ CI: $0.79-1.0$, $P=0.05)$ results. Additionally, men were more likely to indicate a high preference for the return of germ-line sequencing results than women (92 vs. $76 \%$, adjusted RR: $1.06,95 \%$ CI: $1.00-1.12$, $P=0.04)$.

\section{DISCUSSION}

We examined the implementation of paired somatic and germ-line WES at a comprehensive cancer center. We found that although most oncologists have ample experience using and interpreting somatic genomic tests, they have little experience with germ-line testing. Nevertheless, respondents intended to disclose most WES results from both somatic and germ-line testing to patients. Oncologists also expressed concerns about data interpretation, disclosing noncancer findings, and determining the "actionability" of alterations. We also found that patients with advanced lung and colorectal cancer have favorable attitudes toward having a genetic test but moderately low levels of genetic knowledge, and that most want to learn all WES results. Our findings advance the field by demonstrating that although physicians anticipate many challenges to delivering care involving large-scale sequencing, patients with
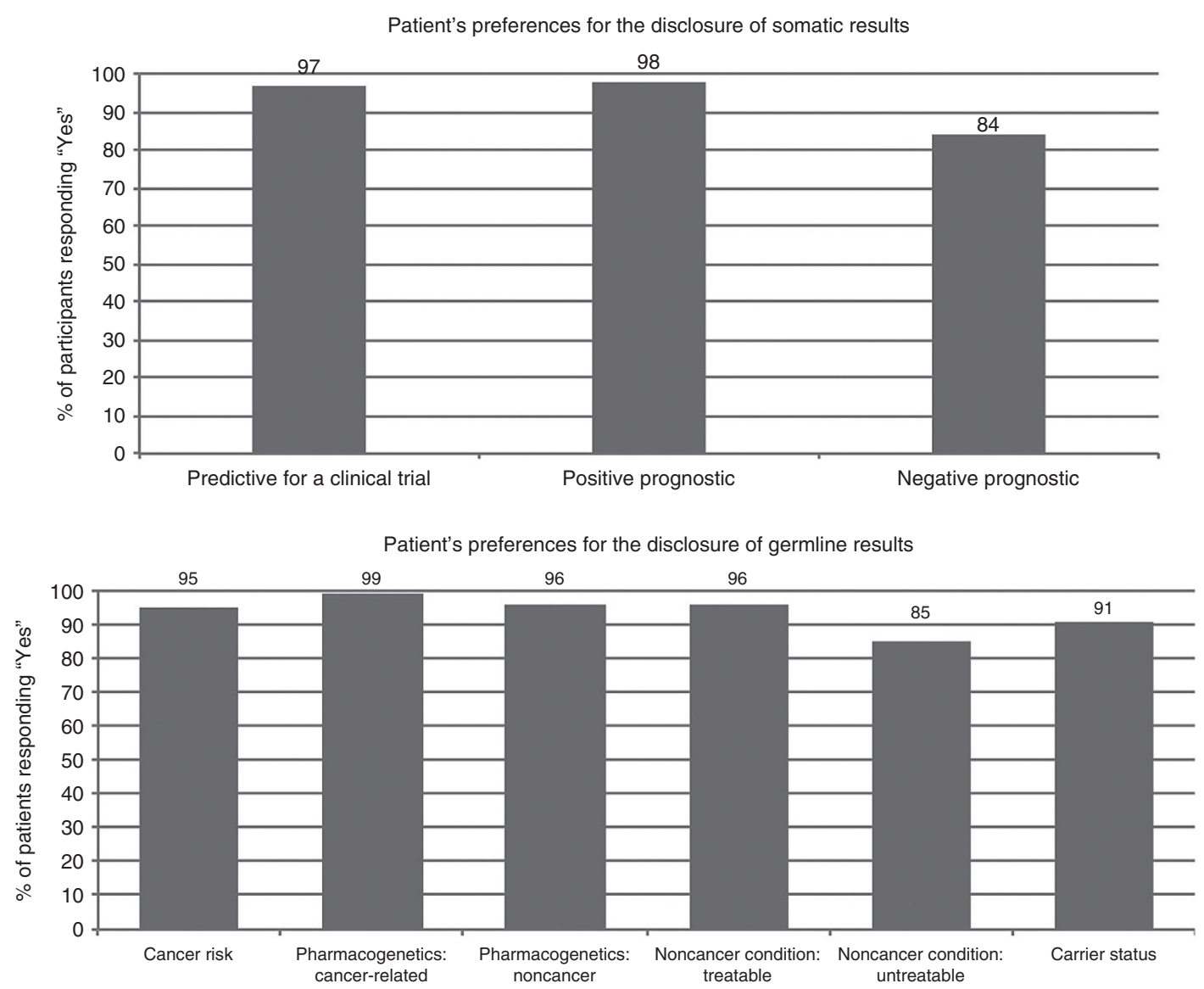

Figure 2 Patients' preferences for the return of somatic and germ-line WES results. 
incurable cancer express a strong desire to learn about genomic findings whether or not they have relevance to their immediate medical care.

When queried about somatic genomic testing, most oncologists were moderately or very confident in their ability to interpret somatic test results in their disease area, explain somatic genomic concepts to patients, make treatment recommendations based on somatic genomic information, and identify appropriate consultants. The high levels of confidence may relate to the fact that the oncologists in our study order and interpret large numbers of somatic tests and that lung and colorectal adenocarcinoma are malignancies in which genomic testing is part of guideline-based cancer care. ${ }^{29,30}$ Nevertheless, oncologists anticipated a number of challenges to delivering somatic WES care, including dealing with and interpreting large volumes of data and determining the actionability of somatic findings. ${ }^{31}$ Some oncologists expressed concerns about how to determine how much somatic data should be shared with patients and how to manage patients' expectations. In addition, oncologists expressed concerns about their ability to keep up with the literature in this rapidly evolving field.

Most oncologists planned to disclose all types of somatic WES findings to their patients with metastatic disease, assuming that patients desired WES information, that they had good performance status, and that a clinically certified laboratory performed the sequencing. Oncologists' responses during qualitative interviews help explain these findings. All interviewees planned to disclose somatic findings if there were an approved targeted therapy for another cancer type or if a clinical trial were available. Liberal attitudes toward somatic disclosure were tied to oncologists' desires to explore different treatment options and to be able to offer enrollment in clinical trials. Several oncologists also stated that patients have a "right to know" somatic information. ${ }^{32,33}$

Ninety-two percent of our gastrointestinal oncologists had ordered or interpreted germ-line tests during the prior year as compared to $38 \%$ of thoracic oncologists. Because genetic testing for familial colorectal cancer syndromes is integrated into standard practice whereas standards for testing for hereditary lung cancer syndromes are just emerging, gastrointestinal oncologists may be more accustomed to ordering germ-line tests than their thoracic counterparts. In addition, 35\% of all oncologists had ordered or interpreted cancer-related pharmacogenetic tests during the prior year. This use of pharmacogenetic testing is notable because the reported uptake of pharmacogenetic testing generally is estimated to be $\operatorname{low}^{34-36}$ despite the fact that pharmacogenetic tests (e.g., DPYD, UGT1A1) are available, polymorphisms in these genes are associated with drug metabolism, and the Food and Drug Administration includes pharmacogenetic information in the label of drugs, including 5-fluorouracil and irinotecan. ${ }^{37}$ Although oncologists have some experience with ordering and interpreting cancer-related germ-line genomic tests, they have less experience with tests that are unrelated to cancer. ${ }^{38}$
Whereas most oncologists in our study intended to disclose most germ-line WES findings to patients, some expressed reluctance to disclose information about untreatable conditions, pharmacogenetic alterations, and information that would require further counseling and psychosocial support. Some oncologists noted that disclosure of a negative prognosis might facilitate patients' prognostic awareness; however, others reported a hesitance to share this information, particularly if the patient was at the end of life. Based on our interview data, oncologists' reluctance to disclose information seems less related to a desire to "protect" patients from bad news than to a desire to give them information that is relevant to their situation and that is in accordance with their preferences. Many oncologists noted that the "actionability" of the information would be a key determinant of disclosure, and that they would be less willing to disclose information if it did not have implications for cancer therapy or prevention. For germ-line disclosure decisions, a few oncologists said that they may not disclose the information if the patient did not have or did not intend to have children. One might hypothesize that in the setting of advanced cancer, providers' and patients' risk-benefit calculations as they weigh the value of germ-line data and disclosure may differ substantially from that in other settings where the clinical utility of germ-line information for patients may be clearer. Additionally, several oncologists noted a desire to involve a genetic counselor or another provider when disclosing germ-line results. Given the complexity of the results and the familial implications of germ-line findings, institutions that offer WES or WGS may need to make relevant clinical and counseling expertise available to oncologists, patients, and patients' family members.

One of the most pressing questions in oncology is how to best support cancer providers as they integrate large amounts of genomic data into routine cancer care. Efforts to support providers must start with efforts to improve the quality of the data in reference genomic databases and with efforts to optimize bioinformatics algorithms and resources for variant calling and interpretation. In addition, sophisticated approaches to physician education and decision support will be essential. Given the rate at which information about genomic variants changes, dynamic genomic reports and point-of-care physician support will help providers to understand the potential implications of somatic and germ-line variants and to better personalize recommendations. In addition to systems interventions, physicians might also be supported through programs developed by their local institutions. For example, several institutions, including the DFCI, have developed multidisciplinary "genomic tumor boards" where providers can discuss patients in a case-based format, highlighting their genomic or proteomic data, to get feedback or input from colleagues with expertise in medical oncology, molecular biology, clinical trials, pathology, and medical ethics. ${ }^{14,39}$

The strong preference of most patients for return of all categories of somatic and germ-line genomic results and the relatively low levels of genetic knowledge suggest that patients will also need assistance in understanding and making informed 


\section{ORIGINAL RESEARCH ARTICLE}

decisions based on genomic tests. Educational resources and decision aids will be needed at the time of initial consent and sample acquisition so patients can provide informed preferences about the types of results they desire. It will be important for patients undergoing cancer-related WES or WGS to understand basic genetic concepts such as the difference between somatic and germ-line testing, that men can pass germ-line genetic mutations to their children, and that not all germ-line mutation carriers will develop disease. Computer-based education interventions have been shown to improve knowledge in the setting of germ-line cancer genetic counseling and may prove effective in this setting. ${ }^{40}$ In addition, given that providers do not routinely elicit patients' preferences for the return of specific results when they order laboratory tests, more work needs to be performed to determine how to best identify and respect patients' preferences for sequencing findings. One possible solution is to capture patients' preferences for sequencing results on the test requisition, therefore allowing the laboratory to customize result reporting. Finally, resources will be needed at the time of returning results to minimize misunderstanding and ensure that the actions that patients and their family members take are based on evidence and consistent with their values. Given the speed with which genomic testing is entering oncology practice, the development of such resources for patients and families is an urgent priority. ${ }^{2,41}$

The present study has several limitations. First, we conducted our study at a single academic center and restricted enrollment to adult patients with advanced lung and colorectal cancer and to their oncologists, suggesting the need for caution in generalizing our findings to other settings and cancer populations. Given that DFCI has an enterprise-wide, multiplex gene sequencing study underway, the oncologists in our study may use genomic testing more frequently than most oncologists. Second, we assessed oncologists' intentions to disclose WES results, which may or may not correspond to their actual behavior. Third, patients had high levels of education and included few members of racial/ethnic minority groups. Fourth, given the novelty of sequencing in the cancer context, several measures used with both patients and physicians were developed specifically for this study and had not previously been validated. Finally, alternative approaches to preference elicitation or pretest education and counseling might have led patients to make different choices about return of results.

In summary, patients with advanced solid tumors express a strong desire for the return of genomic results, including incidental findings. However, these preferences may not be based on a robust understanding of genetics or of the implications of the findings for patients' or their family members' health and medical care. Furthermore, oncologists who work with these patients express concern about their ability to evaluate, communicate, and make decisions about the broad range of somatic findings that WES will produce, as well as about their ability to address germ-line findings that may result from parallel sequencing. Resources to assist physicians and patients in addressing these concerns represent a pressing priority for the cancer community.

\section{SUPPLEMENTARY MATERIAL}

Supplementary material is linked to the online version of the paper at http://www.nature.com/gim

\section{ACKNOWLEDGMENTS}

This study was supported by National Human Genome Research Institute U01HG006492 and U01 HG007303. S.W.G. is also supported by the American Cancer Society (120529MRSG-11-006-01-CPPB). The authors acknowledge all members of the CanSeq team for their hard work and dedication as well as the oncologists and patients who have participated in the study. S.W.G. and S.J. had full access to all of the data in the study and take responsibility for the integrity of the data and the accuracy of the data analysis.

This study was presented in part at the 2014 American Society of Clinical Oncology Annual Meeting (Abstract 1535) and at the 2014 American Society of Human Genetics Annual Meeting (Session 11). National Institutes of Health ClinicalTrials.gov identifier nct02127359.

\section{DISCLOSURE}

J.G. performs consulting or advisory roles for Novartis (family member), Pfizer, and Sequenom; receives research funding from Myriad Genetics, Novartis, and Pfizer (family member). P.A.J. receives postmarketing royalties from DFCl-owned intellectual property on EGFR mutation testing licensed to Lab Corp. E.M.V.A. is a consultant for Syapse and Roche-Ventana. N.W. is a consultant and stockholder in Foundation Medicine. L.G. is an equity holder and consultant for Foundation Medicine, a consultant and sponsored research for Novartis, and a consultant for Boehringer Ingelheim. S.J. was a member of the Data and Safety Monitoring Board for Genzyme/Sanofi until 12 February 2012. The other authors declare no conflicts of interest.

\section{REFERENCES}

1. Green RC, Berg JS, Grody WW, et al.; American College of Medical Genetics and Genomics. ACMG recommendations for reporting of incidental findings in clinical exome and genome sequencing. Genet Med 2013;15:565-574.

2. Robson ME, Bradbury AR, Arun B, et al. American Society of Clinical Oncology Policy Statement Update: genetic and genomic testing for cancer susceptibility. J Clin Oncol; e-pub ahead of print 31 August 2015.

3. Kauff ND, Satagopan JM, Robson ME, et al. Risk-reducing salpingooophorectomy in women with a BRCA1 or BRCA2 mutation. N Engl J Med 2002;346:1609-1615.

4. Domchek SM, Friebel TM, Singer CF, et al. Association of risk-reducing surgery in BRCA1 or BRCA2 mutation carriers with cancer risk and mortality. JAMA 2010;304:967-975.

5. Machens A, Niccoli-Sire P, Hoegel J, et al. Early malignant progression of hereditary medullary thyroid cancer. N Engl J Med 2003;349:1517-1525.

6. Järvinen HJ, Aarnio M, Mustonen $\mathrm{H}$, et al. Controlled 15-year trial on screening for colorectal cancer in families with hereditary nonpolyposis colorectal cancer. Gastroenterology 2000;118:829-834

7. Piccart-Gebhart MJ, Procter M, Leyland-Jones B, et al.; Herceptin Adjuvant (HERA) Trial Study Team. Trastuzumab after adjuvant chemotherapy in HER2-positive breast cancer. N Engl J Med 2005;353:1659-1672.

8. Druker BJ, Talpaz M, Resta DJ, et al. Efficacy and safety of a specific inhibitor of the BCR-ABL tyrosine kinase in chronic myeloid leukemia. $N$ Engl J Med 2001;344:1031-1037

9. Flaherty KT, Puzanov I, Kim KB, et al. Inhibition of mutated, activated BRAF in metastatic melanoma. N Engl J Med 2010;363:809-819.

10. Mok TS, Wu YL, Thongprasert S, et al. Gefitinib or carboplatin-paclitaxel in pulmonary adenocarcinoma. N Engl J Med 2009;361:947-957. 
11. MacConaill LE, Garcia E, Shivdasani P, et al. Prospective enterprise-level molecular genotyping of a cohort of cancer patients. J Mol Diagn 2014;16:660-672.

12. Cheng DT, Mitchell TN, Zehir A, et al. Memorial Sloan Kettering-Integrated Mutation Profiling of Actionable Cancer Targets (MSK-IMPACT): a hybridization capture-based next-generation sequencing clinical assay for solid tumor molecular oncology. J Mol Diagn 2015;17:251-264.

13. Van Allen EM, Wagle N, Levy MA. Clinical analysis and interpretation of cancer genome data. J Clin Oncol 2013;31:1825-1833.

14. Roychowdhury S, Iyer MK, Robinson DR, et al. Personalized oncology through integrative high-throughput sequencing: a pilot study. Sci Trans/ Med 2011;3:111ra121.

15. Stadler ZK, Schrader KA, Vijai J, Robson ME, Offit K. Cancer genomics and inherited risk. J Clin Oncol 2014;32:687-698.

16. Gray SW, Hicks-Courant K, Cronin A, Rollins BJ, Weeks JC. Physicians' attitudes about multiplex tumor genomic testing. J Clin Oncol 2014;32:1317-1323.

17. Nippert I, Harris HJ, Julian-Reynier C, et al. Confidence of primary care physicians in their ability to carry out basic medical genetic tasks-a European survey in five countries-part 1. J Community Genet 2011:2:1-11.

18. Michie S, di Lorenzo E, Lane R, Armstrong K, Sanderson S. Genetic information leaflets: influencing attitudes towards genetic testing. Genet Med 2004;6:219-225.

19. Sanderson SC, Wardle J, Michie $S$. The effects of a genetic information leaflet on public attitudes towards genetic testing. Public Underst Sci 2005;14:213-224.

20. Singer $E$, Antonucci T, Van Hoewyk J. Racial and ethnic variations in knowledge and attitudes about genetic testing. Genet Test 2004;8:31-43.

21. Furr LA, Kelly SE. The Genetic Knowledge Index: developing a standard measure of genetic knowledge. Genet Test 1999;3:193-199.

22. Fagerlin A, Zikmund-Fisher BJ, Ubel PA, Jankovic A, Derry HA, Smith DM. Measuring numeracy without a math test: development of the Subjective Numeracy Scale. Med Decis Making 2007;27:672-680.

23. Chew LD, Griffin JM, Partin MR, et al. Validation of screening questions for limited health literacy in a large VA outpatient population. J Gen Intern Med. 2008;23:561-566.

24. Oken MM, Creech RH, Tormey DC, et al. Toxicity and response criteria of the Eastern Cooperative Oncology Group. Am J Clin Oncol 1982;5:649-655.

25. Basch E, Artz D, Dulko D, et al. Patient online self-reporting of toxicity symptoms during chemotherapy. J Clin Oncol 2005;23:3552-3561.

26. Aaronson NK, Ahmedzai S, Bergman B, et al. The European Organization for Research and Treatment of Cancer QLQ-C30: a quality-of-life instrument for use in international clinical trials in oncology. J Natl Cancer Inst 1993;85:365-376.

27. Degner $L F$, Kristjanson $L J$, Bowman $D$, et al. Information needs and decisional preferences in women with breast cancer. JAMA 1997;277:1485-1492.

28. Zou G. A modified poisson regression approach to prospective studies with binary data. Am J Epidemio/ 2004;159:702-706.
29. NCCN. National Comprehensive Cancer Network Clinical Practice Guidelines in Oncology (NCCN Guidelines $®$ )Non-Small Cell LungCancer Version 3.2014. http://www.nccn.org/professionals/physician_gls/pdf/nscl.pdf. Published 24 January 2014. Accessed 1 February 2014.

30. National Comprehensive Cancer Network Clinical Practice Guidelines in Oncology (NCCN Guidelines ${ }^{\circledR}$ ) Colon Cancer Version 2.2015. J Nat/ Compr Canc Netw 2014:1-143. http://www.nccn.org/professionals/physician_gls/pdf/ colon.pdf. Accessed 16 September, 2015.

31. Van Allen EM, Wagle N, Stojanov P, et al. Whole-exome sequencing and clinical interpretation of formalin-fixed, paraffin-embedded tumor samples to guide precision cancer medicine. Nat Med 2014;20:682-688.

32. Ramoni RB, McGuire AL, Robinson JO, Morley DS, Plon SE, Joffe S. Experiences and attitudes of genome investigators regarding return of individual genetic test results. Genet Med 2013;15:882-887.

33. Sheehan M. The right to know and genetic testing. J Med Ethics 2015;41: 287-288

34. Johnson JA. Pharmacogenetics in clinical practice: how far have we come and where are we going? Pharmacogenomics 2013;14:835-843.

35. Scott SA. Personalizing medicine with clinical pharmacogenetics. Genet Med 2011;13:987-995

36. Relling MV, Gardner EE, Sandborn WJ, et al.; Clinical Pharmacogenetics Implementation Consortium. Clinical Pharmacogenetics Implementation Consortium guidelines for thiopurine methyltransferase genotype and thiopurine dosing. Clin Pharmacol Ther 2011;89:387-391.

37. Center for Drug Evaluation, Research. Genomics - Table of Pharmacogenomic Biomarkers in Drug Labeling. http://www.fda.gov/Drugs/ScienceResearch/ ResearchAreas/Pharmacogenetics/ucm083378.htm. Accessed 23 January 2015.

38. Parsons DW, Roy A, Plon SE, Roychowdhury S, Chinnaiyan AM. Clinical tumor sequencing: an incidental casualty of the American College of Medical Genetics and Genomics recommendations for reporting of incidental findings. J Clin Oncol 2014;32:2203-2205.

39. Parker BA, Schwaederlé M, Scur MD, et al. Breast cancer experience of the molecular tumor board at the University of California, San Diego Moores Cancer Center. J Oncol Pract 2015;11:442-449.

40. Green MJ, Peterson SK, Baker MW, et al. Effect of a computer-based decision aid on knowledge, perceptions, and intentions about genetic testing for breast cancer susceptibility: a randomized controlled trial. JAMA 2004;292: 442-452.

41. Robson ME, Storm CD, Weitzel J, Wollins DS, Offit K; American College of Medical Genetics and Genomics. American Society of Clinical Oncology policy statement update: genetic and genomic testing for cancer susceptibility. J Clin Oncol 2010;28:893-901. 